\title{
Performance analysis of higher mode spoof surface plasmon polariton for terahertz sensing
}

\author{
Haizi Yao ${ }^{1}$, Shuncong Zhong ${ }^{1,2,3}$ a), Wanli Tu \\ 1 Laboratory of Optics, Terahertz and Non-Destructive Testing, School of Mechanical \\ Engineering and Automation, Fuzhou University, Fuzhou 350108, P. R. China \\ ${ }^{2}$ Department of Naval Architecture, Ocean and Marine Engineering, University of Strathclyde, \\ Glasgow G4 OLZ, United Kingdom \\ ${ }^{3}$ Fujian Key Laboratory of Medical Instrument and Pharmaceutical Technology, Fuzhou 350108, \\ P.R. China
}

We investigated the spoof surface plasmon polaritons (SSPP) on 1D grooved metal surface for terahertz sensing of refractive index of the filling analyte through a prismcoupling attenuated total reflection setup. From the dispersion relation analysis and the finite element method-based simulation, we revealed that the dispersion curve of SSPP got suppressed as the filling refractive index increased, which cause the coupling resonance frequency redshifting in the reflection spectrum. The simulated results for testing various refractive index demonstrated that the incident angle of terahertz radiation has a great effect on the performance of sensing. Smaller incident angle will result in a higher sensitive sensing with a narrower detection range. In the meanwhile, the higher order mode SSPP-based sensing has a higher sensitivity with a narrower detection range. The maximum sensitivity is $2.57 \mathrm{THz} / \mathrm{RIU}$ for the second-order mode sensing at 45 degrees internal incident angle. The proposed SSPP-based method has great potential for high sensitive terahertz sensing.

\section{INTRODUCTION}

In recent years there has been an increasing interest in surface plasmonics. One of the most important reason is that as a form of collective oscillation of surface electric charge on metal surface, the surface plasmon polariton (SPP) wave has its unique properties, for instance, SPP has the ability to spatially confine the electromagnetic wave within subwavelength and dramatically enhanced the electromagnetic field intensity in a confined region ${ }^{1}$. Besides, the characteristics of SPP wave is highly related to the outside electric environment contacting the metal surface, in other words, SPP is sensitive to the analyte adhered to metal surface. Therefore SPP wave is often utilized for sensing known as surface plasmon resonance (SPR) sensing technique ${ }^{2,3}$. Especially in the applications of biosensing, SPR sensing is a powerful method to sensitively monitor the dynamic reaction process of biological molecules ${ }^{4,5}$. For many macromolecules have their "finger spectrum", i.e., their respective vibration frequency, locating in the terahertz regimes ${ }^{6-8}$; therefore, terahertz SPR sensing has inherent advantages for biosensing. However, in terahertz regime most metals do not support the highly confined surface plasmon polariton wave because metals resemble a perfect electric conductor for their volume plasma frequency always stand in ultraviolet frequency. Therefore, the advantages of SPP cannot be directly utilized in terahertz region. Fortunately, Pendry et al. theoretically demonstrated that this problem can be overcome by tailoring the metal surface that make the structured surface support a kind of mimicked SPP in terahertz

a) Author to whom correspondence should be addressed. Electronic mail: zhongshuncong@hotmail.com. 
frequency, termed of spoof SPP (SSPP) ${ }^{9,10}$. The structured surface has a lower effective surface plasma frequency and the propagating mode of SSPP wave at lower frequency that is similar to real SPP wave. Commonly, the size of microstructure supporting terahertz SSPP wave is tens of micrometer which is of subwavelength compared to typical terahertz wavelength ( $\lambda=300 \mu \mathrm{m}$ at $f=1 \mathrm{Thz}$ ). The most remarkable is that such SSPP is dependent on the geometry of the structure on metal surface, so it can be arbitrary controlled by designing the geometrics ${ }^{11,12}$. Taking advantage of this feasibility, except for the uniform grooves ${ }^{13}$ several novel plasmonic surface decorated with 1D or 2D structure have been proposed in terahertz frequencies, such as penetrated with trapezoid-shaped slot grooves ${ }^{14}$, textured by square arrays of metallic pillars ${ }^{15}$, composite-periodic gratings ${ }^{16}$ etc. Among these spoof plasmonic metal surface, the case with array of grooves has been investigated most frequently for its manufactural simplicity and typicality. Theoretical and experimental studies showed that such 1D grooved metal surface are capable of confining and guiding the terahertz radiation with a lowered effective surface plasma frequency, and the dispersion relation of such spoof surface propagating wave is mainly dependent on the groove depth ${ }^{17,18}$. This effective lowered surface plasma frequency of structured metal surface offer the possibility to realize the terahertz SPR sensing.

Commonly, SPR sensing system working in visible light frequency range has two schemes which are called Otto and Kretschmann attenuated total reflection coupling (ATR) setups ${ }^{19,20}$. The main difference between two configurations is whether the thin metal film, used to support the SPP wave, is spaced or contacted with the coupling prism bottom. Because Otto setup require precisely controlling of gap distance within one wavelength range whereas Kretschmann system do not, therefore the Kretschmann-based SPR sensing setup is often adopted in the practical applications. The situation may be changed in terahertz SPR sensing because the terahertz wavelength is comparatively large, hundreds of micrometers long, and those strict requirements for the past technical level such as micrometer-level precious tuning can be easily achieved with sate of the art micro-control facilities. Therefore, the Ottobased SPR sensing setup can be put into practice in terahertz regimes. Combined with the Otto prismcoupling configuration, the unique SSPP infuse the new blood to terahertz SPR sensing. Recently, $\mathrm{Ng}$ et al. reported the experimental verification that array-grooves corrugated metal surface can serve as the high sensitive terahertz SPR sensing platform ${ }^{21,22}$. But they only discuss the shallow groove case which only support the fundamental SSPP wave. Actually when the depth of penetrating groove is deep enough there exists the high-order spoof SPP modes ${ }^{17}$. In this paper, we conducted a further discuss about the performance of the high modes SSPP in terahertz SPR sensing. Theoretical and simulated results showed that the higher mode SSPP can offer a more sensitive sensing for the refractive index of the filling analyte, but it has a narrower detection range. Our findings demonstrate that the high mode spoof SPP has the potential to achieve super-sensitive terahertz sensing.

\section{SPR SENSING SYSTEM}

The proposed structure of terahertz SPR sensing was shown in Fig. 1. It consisted of a coupling prism with high refractive index $n_{p}$ and a metal film engraved by a 1D groove array with depth $h$, width $w$, and lattice constant $p$. The metal was beneath the prism bottom forming a gap of $g$. The groove and gap was filled with the analyte with refractive index of $n_{d}$. A collimated terahertz beam was injected through the left facet of prism and then refracted to the bottom with an incident angle $\theta_{\text {int }}$. After interacting with the filling analyte inside gap and grooves, the modified terahertz radiation reflect and exit to the other facet of prism, where the terahertz radiation could be detected. When the momentum 
matching condition is fulfilled, i.e., $k_{\text {spp }}=k_{/ /}$(where $k_{/ /}=k_{0} n_{p} \sin \left(\theta_{\text {int }}\right)$ is the parallel component of wavevector in prism and $k_{\text {spp }}$ is the wavevector of SSPP wave), the corresponding frequency component will coupled into the propagating attenuated SSPP wave forming the resonance. The collected reflectivity spectrum will exhibit a sharp dip at this frequency accordingly. Since the sensitive characteristics of spoof SPP, the coupling frequency will shift as the filling refractive index varies. We can retrieve the value of $n_{d}$ by analyzing data of the shifted reflectivity spectrum with calibrations.

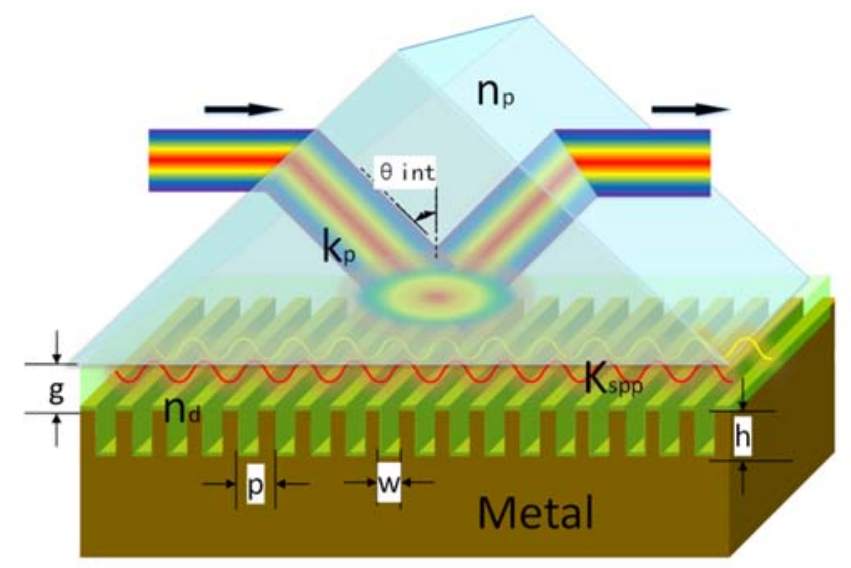

FIG. 1. The prism-coupling based Otto attenuated total reflection (ATR) setup for SPR sensing, comprising a high refractive index prism and a spaced periodically grooved metal film. The grooves and gap are filled with the analyte. Grooves are of width $w$, depth $h$, and periodicity p. $g$ is the distance of the gap between the prism base and the surface of the metal film. $k_{p}$ are the wavevectors in the prism and $k_{\text {spp }}$ is the wavevector of the spoof surface plasmon wave. $\theta_{\text {int }}$ is the internal incident angle at the base of the prism. $n_{p}$ and $n_{d}$ are the refractive index of prism and the filling analyte, respectively.

\section{THEORETICAL ANALYSIS of SPR SENSING SYSTEM}

Under Within the limit of $p<<\lambda_{0}$, the dispersive relation of the supported spoof SPP wave propagating along groove-corrugated surface can be expressed as ${ }^{17}$ :

$$
\cot \left(k_{d} h\right)=\frac{w}{p} \sum_{n} \frac{k_{d}}{q_{z}^{(n)}} s_{n}{ }^{2}
$$

where $k_{d}=2 \pi n_{d} f / c$ is the wavevector of light in a medium with refractive index $n_{d}$; $k_{x}^{n}=k_{s p p}+n_{d} 2 \pi n / p, q_{z}^{(n)}=\sqrt{\left(k_{x}^{n}\right)^{2}-\left(k_{d}\right)^{2}}, s_{n}=\sin c\left(k_{x}^{n} w / 2\right), k_{s p p}$ is the propagation constant for spoof SPP wave and $n$ is the diffraction order. From Eq. (1) we can see that the dispersion relation is related to the structures parameters (e.g., $h, w$ and $p$ ). In fact, the dispersion is primarily dependent on the groove depth $h^{10,12}$. When $h$ is large enough, i.e., $h>m p$ (integer $m$ is the mode order), the high modes of spoof SPP with order $m$ begin to be supported; whilst for the case of $h<p$, there is only the fundamental mode SSPP on the corrugated metal surface ${ }^{17}$.

To demonstrate the influence of groove depth on characteristics of SSPP, we calculated the dispersion relations of SSPP wave supported on the periodical grooves by using Eq. (1). The dispersion curves in first Brillioun zone for $w=0.5 p, h=2.8 p$ and $h=0.5 p$ are respectively plotted in Fig. 2 . In the calculation, filling analyte was assumed to be vacuum with the refractive index of $n_{d}=1.0$. The scatter data are obtained by finite element method by using COMSOL MULTIPHYSICS software. The numerical result is highly agreed with the analysis at fundamental mode but with a considerable deviation at high modes, that is because the theoretical mode in Eq. (1) only consider the fundamental mode in field expansion in groove and high-order modes are ignored. Besides, it is clear that the dispersion curve for the case of $h=0.5 p$ has only one section which is the fundamental guide mode of SSPP wave; whereas, in the case of $h=2.8 p$ the dispersion relation is three-layered which are corresponding to the fundamental 
mode and the higher modes. Insets are the electric field distribution of different modes SSPP at $k_{s p p}=\pi / p$ for the case of $h=2.8 p$. The appearance of zero, one or two nodes of field distribution along vertical direction inside groove prove that they are the zero-order (fundamental mode), first-order and second-order mode of SSPP, respectively. This result is consistent with the prediction from the abovementioned criteria of existence of high mode, i.e., the supported maximum mode order of SSPP is less than three at $h=2.8 p<3 p$. For all modes, the wave vector increase and the dispersion curves get flat as frequency approach its corresponding effective surface plasma frequency. The effective surface plasma frequency is also called the cutoff frequency because only the SSPP wave with a frequency below it can propagate. It is obvious that as to the same fundamental mode, the dispersion relation curve of deep groove case $(h=2.8 p)$ is more suppressed compared to that of shallow groove case ( $h=0.5 p)$, thus, leading to a lower cutoff frequency. The adjustability of SSPP by geometry structure demonstrate that such spoof SPP wave has an significant design flexibility that the effective plasma frequency can be designed across frequency of interest. That is very important to biosensing.

The purpose of this work is to investigate the performance of high modes of SSPP for terahertz sensing, so we only discuss the case of deep groove here. In Fig. 2 the straight dashed line is dispersion relation of vacuum light and the straight dot and dot-dashed lines are the parallel coupling wavevector $k_{/ /}$in prism with internal incident angle of 45 degrees and 60 degrees. In the whole work discussed here, prism is assumed made of $\mathrm{K} 9$ glass with $n_{p}=1.5163$. With a proper $\theta_{\text {int }}$ the $k_{/ /}$line intersect the dispersion curves of the SSPP modes forming intersections, for instance, which are indicated as dots A, $\mathrm{B}$ and $\mathrm{C}$ at $\theta_{\text {int }}=45^{\circ}$. The intersection points determine the expected resonance frequencies $f_{\text {res }}$ at which the incident THz radiation will couple into SSPP wave. In general, the dispersion curves is lower as $n_{d}$ increases. That cause the resonance frequencies redshift which is used as the readout signal for sensing the change of filling refractive index.

The SSPP wave is a kind of slow wave and its effective propagating constant is always larger than the embedding dielectric constant, i.e., $k_{\text {spp }} / k_{0}>n_{d}$. In the case of a certain incident parallel wavevector, $k_{/ /}=k_{0} n_{p} \sin \left(\theta_{\text {int }}\right)$, considering the momentum matching condition, $k_{/ /}=k_{\text {spp }}$, it is obviously concluded that $n_{d}$ should be less than $n_{p} \sin \left(\theta_{\text {int }}\right)$. In other words, only when the $k_{/ /}$line is at right of the light line of dielectric, intersecting with the dispersion line of SSPP, there will be the resonance coupling, i.e., the sensing signal. Therefore, for a configuration with a certain prism and incident angle, the theoretical maximum detection range of $n_{d}$ is limited to $n_{p} \sin \left(\theta_{\text {int }}\right)$. However, the large value of $n_{p} \sin \left(\theta_{\text {int }}\right)$ is not better because when it is too large the line of $k_{/ /}$excessively deviate from the light line and beyond the right "boundary" of dispersion curves. As a result, there will be no intersection in the first Brillioun zone, i.e., the resonance will not occur. Therefore, the practical maximum detectable refractive index of different SSPP mode sensing are also limited by their respective "boundary" frequencies at the vertical edge of $k_{s p p}=\pi / p$.

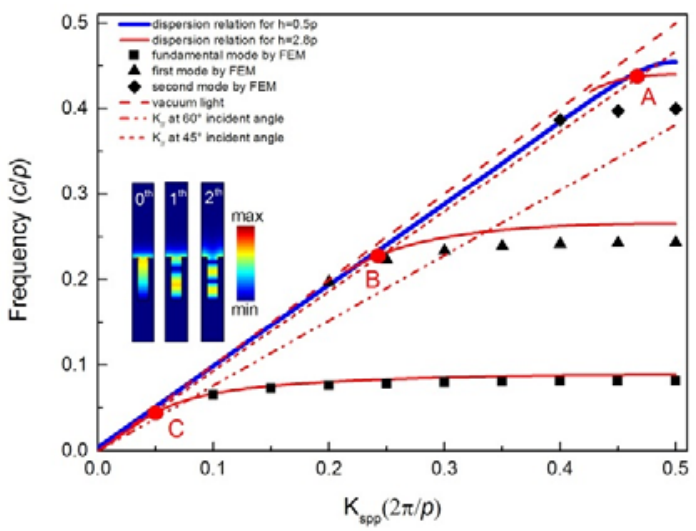

FIG. 2. Dispersion relation of the different mode SSPP on the grooved metal surface. The blue bold solid line is for the case of $h=$ $0.5 p$ and the red solid curve lines are for different mode SSPP in case with $h=2.8 p$. From bottom to top are corresponding to the zero-order, first-order and second-order modes. In two case the width of groove $w$ is assumed equal to $0.5 p$. The straight dashed line is the dispersion relation of the light and the straight dot and dot-dashed lines are the dispersion relations of the parallel wavevector $k_{/ /}$with internal incident angle of 45,60 degrees, respectively. The scatter data (rectangle, triangle, diamond) are the numerical results using finite element method. The dots A,B and C are the intersections between different mode SSPP dispersions and coupling wavevector $k_{/ /}$at 45 degrees incident angle. The insets show the electric field distributions for different modes of SSPP in case of $h=2.8 p$ at $k_{\text {spp }}=\pi / p$. 
From Fig. 2 it was seen that the different modes dispersion curves are separated with each other; also, except for the fundamental mode, every high mode SSPP has a respective start-off frequency at which dispersion curve intersect with the light line, and also a "boundary" frequency accordingly. The higher "boundary" frequency offer a narrower detection range for the higher mode-based sensing. For the case of deep groove, the fundamental mode dispersion is below that of the higher mode, thus, the line passing the origin and "boundary" of fundamental mode has a larger titled angle than that of higher modes. That is to say, the lower mode SSPP-based sensing can support a larger $n_{p} \sin \left(\theta_{\text {int }}\right)$, thus, a broader detection range than higher mode.

Except for the difference in detection range, the sensing sensitivity of different mode-based sensing is also different. The sensitivity is defined as the resonance frequency-shift dividing by the refractive index change. Due to the more confinement of electric field in high mode, the high modes SSPP is much more susceptible to the change of outside electric environment. Fig. 3 displays the trend of the dispersion relation curves with different filling media of $n_{d}=1.0,1.15$, and 1.3, indicated by the solid, dashed and dot-dashed plots, respectively. The geometry parameters are set as: $p=60 \mathrm{um}, w=0.5 p$, and $h=2.8 p$. For each plot in Fig. 3, the straight tangential line on the left represents the light line in its corresponding dielectric environment, i.e., $k_{d}=n_{d}(2 \pi f / c)$, and the curve on the right is the dispersion relation of spoof surface plasmon polaritons wave. The dispersion lines of propagating SSPP wave locating at the right of the corresponding light lines indicate they are a kind of slow and confined wave. It can be seen, as the $n_{d}$ increase the dispersions of all modes are suppressed and right-shifted, which will lead to the intersections slipping along the $k_{/ /}$line to a lower positions (refer to Fig. 2). The change of the intersection frequencies with increasing $n_{d}$, indicated by $\Delta$, will be presented as the shift of the minimum dip in reflectivity spectrum. Obviously, the higher mode dispersion being suppressed more than the lower mode lead a larger $\Delta$ implying that the higher mode SSPP-based sensing has a higher sensitivity than lower mode.

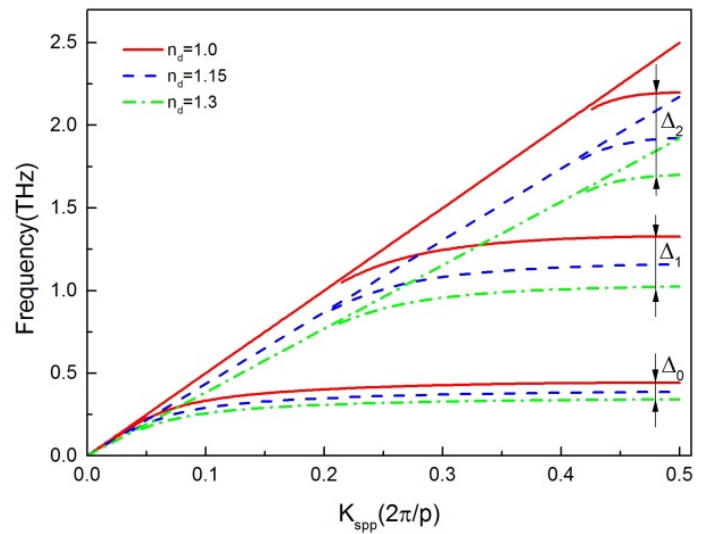

FIG. 3 The influence of increase of filling refractive index to the dispersion relations of different mode SSPP for case of $h=2.8 p$. The curves from top to bottom are corresponding to $n_{d}=1.0,1.15$, and 1.3, respectively. The three left straight lines are the corresponding light lines. $\Delta$ is used to indicate the change of dispersion curves for different mode SSPP.

To have a further insight into the relation of resonance frequencies and filling refractive index, we plotted the resonance frequencies against $n_{d}$ by replacing the term of $k_{s p p}$ in Eq. (1) by $k_{/ /}$. The calculated theoretical resonance frequencies functioned by $n_{d}$ are plotted in Fig. 4 . The solid lines and dashed lines are corresponding to a small parallel wavevector in prism $\left(k_{/ /}=1.5163 \times \sin \left(45^{\circ}\right)=1.07\right)$ and a large one $\left(k_{/ /}=1.5163 \times \sin \left(60^{\circ}\right)=1.31\right)$, respectively. The separated three lines for each coupling case in figure are corresponding to the three different modes sensing. It can be seen all the resonance frequencies decrease as $n_{d}$ increase approaching to the limit of $n_{d}=n_{p} \sin (\theta)$. This phenomenon is expected because the SPR frequencies, indicated by dots in Fig. 2, will slip toward to the lower tangency points between dispersion curves and light lines. The nearly straightness of these lines facilitate the high linearity of this SSPP based sensing.

Unlike the case of small parallel wavevector in which the minimal detectable $n_{d}$ for all mode sensing start at $n_{d}=1.0$ shown as solid lines in Fig. 4, the minimal detectable $n_{d}$ of the second modebased sensing at large $n_{p} \sin (\theta)$ has a start-off value indicated by the a star symbol. This is because when 
the incident angle is large, the related $k_{/ /}$line is below the "boundary" of second mode dispersion curve of $n_{d}=1.0$ as shown in Fig. 2. Thus, no resonance coupling occur at small $n_{d}$. Only when the dispersion curves are lowered enough with an increased $n_{d}{ }^{0}$, it begin to cross with the $k_{/ /}$line and the coupling of evanescent wave to SSPP wave begin to be supported. Therefore, for a large incident angle the detectable refractive index range of high mode SSPP-based sensing is narrowed, i.e., from $n_{d}{ }^{0}$ to $n_{p} \sin (\theta)$.

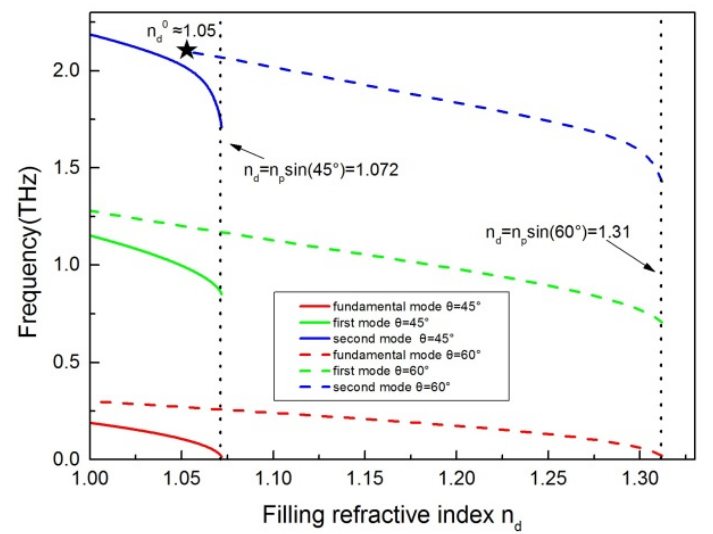

FIG. 4 Theoretic resonance frequencies functioned by filling refractive index in cases of a small and a large incident angle. The solid and dashed lines are for $\theta_{\text {int }}=45^{\circ}$ and $\theta_{\text {int }}=60^{\circ}$, respectively. The star symbol indicate the minimal detectable value for the second mode SSPP sensing in the case of a large incident angle. The two vertical dot lines are used to indicate the maximum detection limit.

It is also obvious that the theoretical resonance frequencies for higher mode has a steeper drop than that for the lower mode, which mean the higher mode SPR sensing has a larger sensitivity that is consistent with the prediction in Fig. 3. Besides, as to the same mode sensing, the decrease in resonance frequencies of a smaller incident angle has a larger gradient than that of large incident angle, which imply that a small $k_{/ /}$input can offer an improved sensitive sensing. This is because the dispersion curve of such mimicked SPP behave a dramatic increase at the region close to light line and maintain almost flat at far away from light line, thus, the large gradient of dispersion curve close to the light line will offer a large vertical slipping distance of the intersections along the fixed $k_{/ /}$line. Therefore, for the smaller incident angle the resonance frequency, close to light line, is more sensitive to the change of the dispersion. It is worth to mention that the improvement of sensitivity is based on the sacrifice of the detection band limited by the small incident coupling wavevector.

All in all, the higher mode-based sensing has a higher sensitivity but a narrower detection range of filling refractive index; similarly, the configuration with a small incident angle possess an improved sensitivity but also a narrowed maximum detection range.

\section{SIMULATION AND RESULTS}

In order to examine the theoretical analysis discussed previously, we calculated the reflectivity of different modes SPR sensing through 2D numerical simulation using COMSOL MULTIPHYSICS software. The simulation schematic graph is shown as the inset in Fig. 5(a). In the simulation, metal was supposed to be Au described by Drude mode with plasma frequency $\omega_{p}=1.2 \times 10^{16} \mathrm{~Hz}$ and scattering frequency $\gamma=5.23 \times 10^{13} \mathrm{~Hz}^{23}$. Periodic boundary is applied to the propagating direction. The TMpolarized terahertz wave is incident from the upper port with oblique angle $\theta_{\text {int }}$. The period, width, and depth of periodic groove are set as the mentioned above. For consistent with above theoretical analysis, the incident angle were set as 45 degrees and 60 degrees to represent the cases of small and large parallel evanescent wavevector, respectively. Note that the selected incident angle $\theta_{\text {int }}$ should be larger than the critical angle $\theta_{\mathrm{c}}=\arcsin \left(1 / \mathrm{n}_{p}\right)=41^{\circ}$ to convert the incident radiation to evanescent wave at the prism bottom for a better coupling. In the case of small incident angle, the $k_{/ /}$line cross the three mode dispersion relation curves at $n_{d}=1.0$ as shown in Fig. 2, thus the detection range of refractive index for all mode sensing are from $n_{d}=1.0$ to $n_{d}=n_{p} \sin \left(45^{\circ}\right)=1.07$; whereas, for the case of large incident angle, because the $k_{/ /}$line is below the dispersion curves "boundary" of second modes and only cross 
the fundamental and first mode dispersion relation curves at $n_{d}=1.0$, thus, the second modes-based sensing has a greater detectable minimum value of refractive index, $n_{d}{ }^{0}$. Referring to the detectable range for different modes shown in Fig. 4, we choose several representative $n_{d}$ values to test the proposed SPR sensing approach.

The coupling gap has an important influence on the coupling efficiency of evanescent wave into spoof SPP. Commonly, the selected gap distance for the efficient coupling in Otto setup is of one wavelength level ${ }^{24}$ and is varied with electric function of analyte ${ }^{25}$. In this work, the resonance frequency of different mode sensing is different, thus the optimal gap distance for respective mode SPR is different. We obtained the optimal coupling gaps by fitting the reflectivity values at the resonance frequencies along various gap distances adopted (not shown in paper). Through several simulations we obtained the approximated optimal coupling gap distance for different mode SPR sensing for $n_{d}=1.0$ at small incident angle case, $g_{\text {opt }}{ }^{0}=1800 u m, g_{\text {opt }}{ }^{1}=240 u m, g_{\text {opt }}{ }^{2}=140 u m$. For large incident angle case, the three modes-based sensing also has their respective optimal coupling gaps, $g_{\text {opt }}{ }^{0}=400 u m, g_{\text {opt }}{ }^{1}=100 u m, g_{\text {opt }}{ }^{2}=90 u m$. Note that the optimal coupling gap distance for second mode, i.e., $g_{\text {opt }}{ }^{2}=90 \mathrm{um}$, is responsible to the minimal detectable filling refractive index, $n_{d}=1.05$.

Next we evaluated the performance of the different modes SPR sensing via comparing the shift of resonance frequencies caused by the increase of filling refractive index. The reflectivity of different mode SPR sensing for two $n_{p} \sin (\theta)$ are displayed in Fig. 5(a-b), respectively. It is clear that there is a corresponding dip with the minimum reflection for each mode sensing to a certain $n_{d}$. The dipfrequency is the resonance frequency at which the incident radiation couple into the SSPP wave. It also clearly showed that for all mode SSPP sensing the resonance frequencies behave with a redshift as the refractive index $n_{d}$ increases that is consistent with the theoretical prediction: lowered dispersion relation curve resulting from increasing $n_{d}$ lead to a lower intersection, i.e., a red-shifted frequency. Besides, in both figures, the displacement of adjacent dips of higher modes SPR are larger than those of lower modes SPR, verifying the higher mode-based SPR sensing has a higher sensitivity.

However, in all cases the resonance dips get broadened and shallow as the detection refractive index approach to its upper limit. This broadening of dips can be attributed that the increase of $n_{d}$ simultaneously affect the dispersion relation of spoof SPP and the penetration depth into medium of evanescent wave from the base of prism. As the result of increasing $n_{d}$, the resulting less comparison in optics index between the prism and its contacting medium lead a more extension of evanescent field into the coupling gap, and the increased extension will cause an extra reflection at grooved surface. In other words, the adopted approximately optimal gap $g_{\text {opt }}$ only apply to the configuration with the certain initial filling index. Thus, when the filling index depart from the predesigned value, the optimal coupling regime was broken. The more the increased refractive index deviate from the present value the more thoroughly is the optimal coupling regime broken. The broken coupling give rise to the lower coupling efficiency and resulting broad, shallow reflectivity dip. Note that for the reflectance of fundamental mode sensing for $n_{d}=1.3$ in panel (b) it is too shallow to identify the valid resonance, that is likely because the dispersion curve is too close to the light line so that less energy is coupled. It is worth mentioning that the obtained resonance frequencies for increased $n_{d}$ are modified by a small amount of frequencyshift, because the adopted gap distance $g_{\text {opt }}$ are shorter than the real corresponding optimal coupling gap distance for the increased $n_{d}{ }^{25}$. But this frequency-shift is very small so that it can be neglected ${ }^{24}$. 

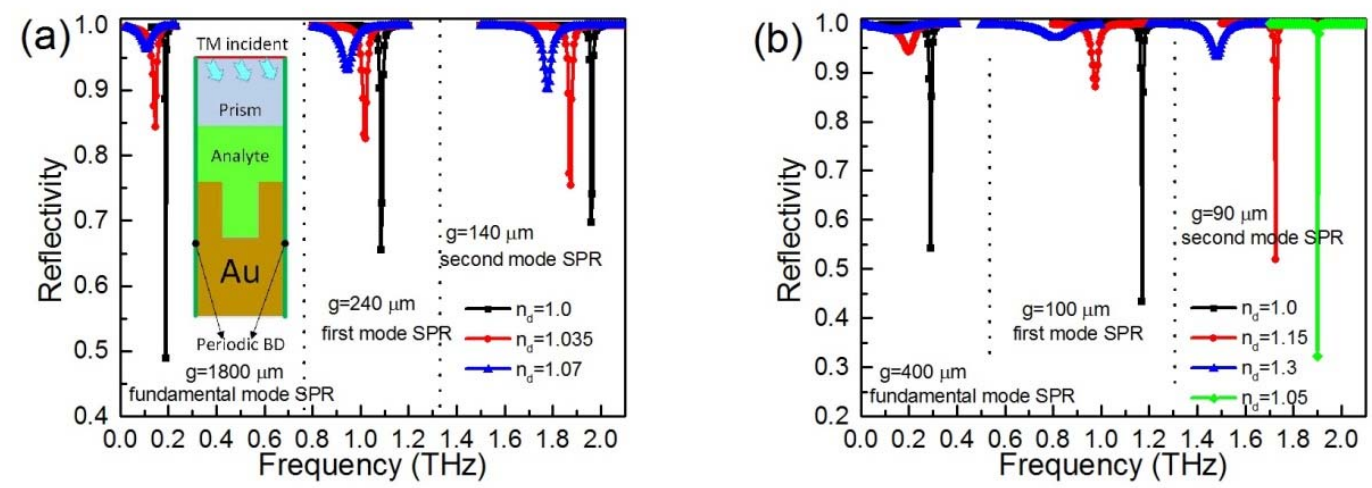

FIG. 5 The reflectivity spectra of different mode SPR sensing at a different incident angle. (a) for internal incident angle $\theta_{\text {int }}$ $=45^{\circ}$; (b) for internal incident angle $\theta_{\text {int }}=60^{\circ}$. The dot lines in figure are for eye-guide to distinguish the different mode SPR. Note the minimal detection refractive index for the second mode-base sensing of large angle input is $n_{d}=1.05$. The inset in panel (a) is the simulation schematic.

\section{COMPARISON OF SENSING SENSITIVITY}

In order to compare the sensitivity of different mode-based sensing, we plotted the resonance frequencies along the refractive indices, as shown in Fig. 6 . The point data were obtained by fitting the dip frequencies in the reflectivity in Fig. 5. The solid lines are the linear fittings. We can see that whether for the fundamental or high modes, the resonance frequencies keep a high linear relation to refractive index, $n_{d}$. The fitting lines for different modes sensing at 45 degrees incident angle are given as $f_{s p 0}=1.33-1.41 n_{d}$ (fundamental mode), $f_{s p 1}=3.08-2.0 n_{d}$ (first-order mode), $f_{s p 2}=4.53-2.57 n_{d}$ (second-order mode), respectively. The fitting lines for the 60 degrees incident angle are given as $f_{s p 0}=0.89-0.60 n_{d}$ (fundamental mode), $f_{s p 1}=2.36-1.20 n_{d}$ (first-order mode), $f_{s p 2}=3.63-1.65 n_{d}$ (second-order mode), respectively. Obviously, whether for either case the higher mode sensing has a larger gradient, i.e., a higher sensitivity. Also, the configuration with a small incident angle has an improved sensitivity compared to that with the large incident angle. Furthermore, we find the sensitivity is roughly proportional to the mode order. For instance, in the case of large incident angle the sensitivity of the second mode( $1.65 \mathrm{THz} / \mathrm{RIU})$ is near three time of that for fundamental mode $(0.6 \mathrm{THz} / \mathrm{RIU})$ and the first mode sensing has a double sensitivity, 1.2 THz/RIU. That is because each propagating mode of SSPP can be considered the transfer of a corresponding modified resonant mode along the surface through coupling between the opening cavities. For a high mode, the electric field inside groove possess a corresponding resonant frequency at which $h \approx m \times \lambda_{s p} / 2$, where $m$ is positive integer and also the mode order. In the case of a certain groove depth, the total resonance length for each mode is fixed. When the outside electric environment change the resonance frequency will shift to maintain the total resonance length, therefore, the change of resonance frequency is proportional to the mode order, $m$. The simulation results are consistent with the theoretical analysis. These sensitivity values for high modes are higher than the results reported previously ${ }^{26,27}$. It is worth mentioning that our simulated sensitivity is far larger than the experimental result from a similar setup, i.e., $0.49 \mathrm{THz} / \mathrm{RIU}$ for nitrogen $\left(n_{d}=1.0\right)^{21}$.

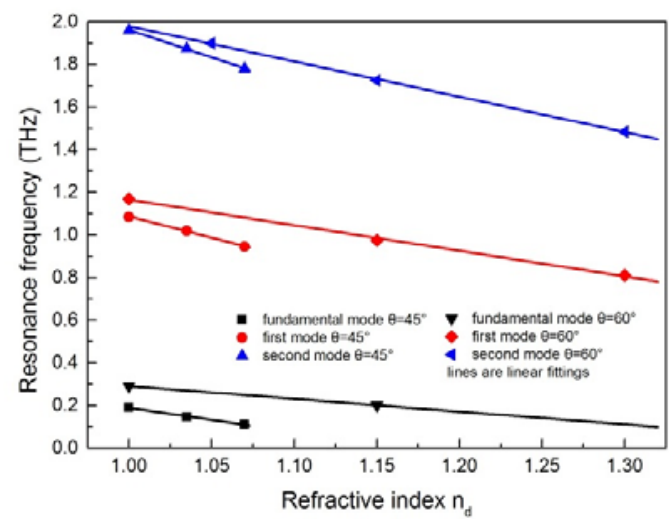

FIG. 6 The resonance frequencies functioned by filling refractive index $n_{d}$ for different mode-based sensing with different incident angle. The solid lines are the linear fittings. 


\section{CONCLUSIONS}

In summary, we have numerically investigated the possibility of refractive index sensing utilizing terahertz spoof surface plasmon polaritons on grooved metal surface. Using the dispersion relation analysis and 2D FEM simulation, it was shown that each propagating mode of SSPP can serve as sensing for refractive index of the filling analyte under a proper coupling gap. Furthermore, we have also demonstrated that the higher mode SPR sensing has a higher sensitivity but a probable narrower detection range for sensing refractive index; also, the small incident angle can offer an improved sensitivity, but it has a limited detection maximum. It was highlighted that the tunable incident angle could be utilized to make the sensing of refractive index locate at region of the high mode to achieve a high sensitivity. Moreover, the response of the spoof SPP can be engineered by designing its geometrical parameters, the sensing for analyte can be conducted in frequency region of interest that is very important to biosensing. In addition to the high sensitivity, the inherent macro groove channels on surface make this kind of spoof SPP-based SPR approach promising for sensing numerous analyte including gaseous and fluidic molecules.

\section{ACKNOWLEDGEMENTS}

We gratefully acknowledge support from the Fujian Provincial Excellent Young Scientist Fund (2014J07007), the National Natural Science Foundation of China (51005077), the Training Program of Fujian Excellent Talents in Universities, the Specialised Research Fund for the Doctoral Program of Higher Education, the Ministry of Education, P. R. China (20133514110008) and the Ministry of Health, P.R. China (WKJ-FJ-27). The authors would like to thank Dr. Yaochun Shen (University of Liverpool, UK) for his suggestions on SPP.

${ }^{1}$ J. M. Pitarke, V. M. Silkin, E. V. Chulkov, and P. M. Echenique, Reports on Progress in Physics 70, 1 (2007).

${ }^{2}$ J. Homola, S. S. Yee, and G. Gauglitz, Sensors and Actuators B: Chemical 54, 3 (1999).

${ }^{3}$ P. Pattnaik, Applied biochemistry and biotechnology 126, 79 (2005).

${ }^{4}$ J. Homola, Chemical Reviews 108, 462 (2008).

${ }^{5}$ J. Homola, Surface plasmon resonance based sensors, Vol. 4 (Springer, 2006)

${ }^{6}$ J.-i. Nishizawa, K. Suto, T. Sasaki, T. Tanabe, and T. Kimura, Journal of Physics D: Applied Physics 36, 2958 (2003).

${ }^{7}$ M. Walther, P. Plochocka, B. Fischer, H. Helm, and P. Uhd Jepsen, Biopolymers 67, 310 (2002).

${ }^{8}$ T. Globus, D. Woolard, T. Khromova, T. Crowe, M. Bykhovskaia, B. Gelmont, J. Hesler, and A. Samuels, Journal of biological physics 29, 89 (2003).

${ }^{9}$ F. Garcia-Vidal, L. Martin-Moreno, and J. Pendry, Journal of optics A: Pure and applied optics 7, S97 (2005).

${ }^{10}$ J. Pendry, L. Martin-Moreno, and F. Garcia-Vidal, Science 305, 847 (2004).

${ }^{11}$ M. Nazarov and J. L. Coutaz, Journal of Infrared, Millimeter, and Terahertz Waves 32, 1054 (2011).

${ }^{12}$ C. R. Williams, S. R. Andrews, S. A. Maier, A. I. Fernández-Domínguez, L. Martín-Moreno, and F. J. García-Vidal, Nature Photonics 2, 175 (2008).

${ }^{13}$ A. Rusina, M. Durach, and M. I. Stockman, Applied Physics A 100, 375 (2010).

${ }^{14}$ F. M. Zhu, Y. Y. Zhang, L. F. Shen, and Z. Gao, Journal of Electromagnetic Waves and Applications 26, 120 (2012).

${ }^{15}$ Z. Gao, L. Shen, J.-J. Wu, T.-J. Yang, and X. Zheng, Optics Communications 285, 2076 (2012).

${ }^{16}$ X. Gao, J. H. Shi, H. F. Ma, W. X. Jiang, and T. J. Cui, Journal of Physics D: Applied Physics 45, 505104 (2012).

${ }^{17}$ T. Jiang, L. Shen, X. Zhang, and L.-X. Ran, Progress In Electromagnetics Research M 8, 91 (2009).

${ }^{18}$ J. B. Pendry, L. Martin-Moreno, and F. J. Garcia-Vidal, Science 305, 847 (2004).

${ }^{19}$ A. Otto, Zeitschrift für Physik 216, 398 (1968).

${ }^{20}$ E. Kretschmann and H. Raether, Zeitschrift Fuer Naturforschung, Teil A 23, 2135 (1968).

${ }^{21}$ B. Ng, J. Wu, S. M. Hanham, A. I. Fernández - Domínguez, N. Klein, Y. F. Liew, M. B. Breese, M. Hong, and S. A. Maier, Advanced Optical Materials 1, 543 (2013).

${ }^{22}$ B. Ng, S. M. Hanham, J. Wu, A. I. Fernández-Domínguez, N. Klein, Y. F. Liew, M. B. Breese, M. Hong, and S. A. Maier, ACS Photonics (2014). 
${ }^{23}$ M. Ordal, L. Long, R. Bell, S. Bell, R. Bell, R. Alexander Jr, and C. Ward, Applied Optics 22, 1099 (1983).

${ }^{24}$ A. OTTO, in The surface polariton response in attenuated total reflection, New York, 1974, p. 117.

${ }^{25}$ H. Yao and S. Zhong, Optics Express 22, 25149 (2014).

${ }^{26}$ B. Ng, S. Hanham, V. Giannini, Z. Chen, M. Tang, Y. Liew, N. Klein, M. Hong, and S. Maier, Optics express 19, 14653 (2011).

${ }^{27}$ B. Reinhard, K. M. Schmitt, V. Wollrab, J. Neu, R. Beigang, and M. Rahm, Applied Physics Letters 100 (2012). 